\title{
The Research on the Methods of Diagnosing the Steam Turbine Based on the Elman Neural Network
}

\author{
Junru Gao, Yuqing Wang \\ Hebei University of Engineering, Handan China.
}

Received 2013

\begin{abstract}
This paper introduces a kind of diagnosis principle and learning algorithm of steam turbine fault diagnosis which based on Elman neural network. Comparing the results of the Elman neural network and the traditional BP neural network diagnosis, the results shows that Elman neural network is an effective way to improve the learning speed, effectively suppress the minimum defects that the traditional neural network easily trapped in, and shorten the autonomous learning time. All these proves that the Elman neural network is an effective way to diagnose the steam turbine.
\end{abstract}

Keywords: Steam Turbine Fault Diagnosis; Elman Neural Network; BP Neural Network

\section{Foreword}

The fault of steam turbine is a complicated system which is nonl near and multivariable identification. If using traditional mathematical model to diagnose its fault, because traditional mathematical model can only reflect linear law of steam turbine's fault. When the construction of system is unknown and nonlinear, the accuracy rate of diagnosing by traditional athematical model is low, which may lead to misdiagnose. As for the complicated relation between the sign and type of steam turbine fault, Elman neural network provide a new way to deal with steam turbine's fault. This paper discusses the method of diagnosing steam turbine's fault based on Elman neural network and BP neural network.. And proved the training and result of these two neural network by doing simulation example, which result in compared with BP neural network, if using Elman neural network, it will be easier to change structural parameter, take shorter training time and have reliable performance, which is better than BP neural network to apply to diagnosing fault.

\section{BP Neural Network Structure and Training}

Due to steam turbine's generator set has a complicated construction system, multiple failure mode and many sign of fault signature. We can diagnose the fault of steam turbine's generator set by analyzing these sign of

Foundation item: natural science funds of Hebei province (F2012 402021), Junru Gao (1969-), female, associate professor, Master Instructor, Mainly engaged in power and electrical engineering teaching and research work. fault signature, and by means of formerly experience, more than $90 \%$ of all kinds of fault of steam turbine can be found by diagnosing vibration fault of steam turbine. This paper refers to the related research, making ten regular fault as analysis object. The form 1 provides frequency spectrum's value distribution of fault signature on $0.01 / 0.39 f 、 0.40 / 0.49 f 、 0.5 f 、 0.51 / 0.99 f 、 f 、 2 f 、 3 / 5 f$ 、 odd times $\mathrm{f}$ and more than $5 \mathrm{f}$, which were given after normalization all ten regular fault information. We want to make it as a training sample for the method of diagnosing neural network model's fault.

Using the data of Table 1 as input sample and Table 2 as output sample to have training. This paper takes 3 layer BP neural network model which has 9 input layer neuron numbers: $\mathrm{x} 1, \mathrm{x} 2, \mathrm{x} 3, \ldots, \mathrm{x} 9$, Inputting the vibration fault information after pretreatment. The number of neurons in the output layer is 10, they are Rotor unbalance(F1), Aerodynamic coupling(F2),shaft misalignment(F3), oil whirl(F4), Rotor adia lub(F4),Symbiotic Loosening(F6), Thrust bearing damage(F7),surge (F8), Bearing pedestal looseness (F9), Range bearing stiffness (F10). According to $m=\sqrt{n_{1}+n_{2}}+\alpha$ and the adjustment after practical training, The number of hidden layer neurons is 12 , Training error is 0.01 , learning rate is 0.2 , excitation function of hidden layer neuron is $\mathrm{S}$ function, The excitation function of the output layer neuronsis purelin function that is linear function, the function of training is TRAINSCG, but the disadvantage of it is when the training is misconvergence, it will stop training, and takes less time than other algorithm.

$\mathrm{n}$ order to explain the application of BP neural network in the fault diagnosis of steam turbine is reasonable, 
Table 1. The sorts of fault diagnosis for steam turbine and results of spectrum analysis.

\begin{tabular}{lccccccccc}
\hline \multicolumn{1}{c}{ Fault samples } & F1 & F2 & F3 & F4 & F5 & F6 & F7 & F8 & F9 \\
\hline frequency range & $\mathbf{0 . 0 1 / 0 . 3 9 f}$ & $\mathbf{0 . 4 0 / 0 . 4 9 f}$ & $\mathbf{0 . 5 f}$ & $\mathbf{0 . 5 1 / 0 . 9 9 f}$ & $\boldsymbol{f}$ & $\boldsymbol{2} \boldsymbol{f}$ & $\mathbf{3 / 5 f}$ & Odd times $\boldsymbol{f}$ & high frequency $>\mathbf{5 f}$ \\
Rotor unbalance & 0.00 & 0.00 & 0.00 & 0.00 & 0.90 & 0.05 & 0.05 & 0.00 & 0.00 \\
Aerodynamic coupling & 0.00 & 0.30 & 0.10 & 0.60 & 0.00 & 0.00 & 0.00 & 0.00 & 0.10 \\
Shaft misalignment & 0.00 & 0.00 & 0.00 & 0.00 & 0.40 & 0.50 & 0.10 & 0.00 & 0.00 \\
Oil whirl & 0.10 & 0.80 & 0.00 & 0.10 & 0.00 & 0.00 & 0.00 & 0.00 & 0.00 \\
Rotor radial rub & 0.10 & 0.10 & 0.10 & 0.10 & 0.20 & 0.10 & 0.10 & 0.10 & 0.10 \\
Symbiotic loosening & 0.00 & 0.00 & 0.00 & 0.00 & 0.20 & 0.15 & 0.40 & 0.00 & 0.25 \\
Thrust bearing damage & 0.00 & 0.00 & 0.10 & 0.90 & 0.00 & 0.00 & 0.00 & 0.00 & 0.00 \\
surge & 0.00 & 0.30 & 0.10 & 0.60 & 0.00 & 0.00 & 0.00 & 0.00 & 0.00 \\
Bearing pedestal looseness & 0.90 & 0.00 & 0.00 & 0.00 & 0.00 & 0.00 & 0.00 & 0.10 & 0.00
\end{tabular}

Table 2. The outputs of ANN fault diagnosis.

\begin{tabular}{lccccccccc}
\hline \multicolumn{1}{c}{ Fault samples } & \multicolumn{7}{c}{ The output of the network layer } & & \\
\cline { 2 - 8 } & $\boldsymbol{F 1}$ & $\boldsymbol{F 2}$ & $\boldsymbol{F 3}$ & $\boldsymbol{F 4}$ & $\boldsymbol{F 5}$ & $\boldsymbol{F 6}$ & $\boldsymbol{F 7}$ & $\boldsymbol{F 8}$ & $\boldsymbol{F 9}$ \\
\hline Rotor unbalance & 1 & 0 & 0 & 0 & 0 & 0 & 0 & 0 & 0 \\
Aerodynamic coupling & 0 & 1 & 0 & 0 & 0 & 0 & 0 & 0 & 0 \\
Shaft misalignment & 0 & 0 & 1 & 0 & 0 & 0 & 0 & 0 & 0 \\
Oil whirl & 0 & 0 & 0 & 1 & 0 & 0 & 0 & 0 & 0 \\
Rotor radial rub & 0 & 0 & 0 & 0 & 1 & 0 & 0 & 0 & 0 \\
Symbiotic loosening & 0 & 0 & 0 & 0 & 0 & 1 & 0 & 0 & 0 \\
Thrust bearing damage & 0 & 0 & 0 & 0 & 0 & 0 & 1 & 0 & 0 \\
surge & 0 & 0 & 0 & 0 & 0 & 0 & 0 & 1 \\
Bearing pedestal looseness & 0 & 0 & 0 & 0 & 0 & 0 & 0 & 0 \\
Range bearing stiffness & 0 & 0 & 0 & 0 & 0 & 0 & 0 & 0 \\
\hline
\end{tabular}

making turbine vibration fault data of document 3 and 4 as the BP neural network calibration sample, that is The input of the neural network is: [0.39 0.07 0.000 .060 .00 $\left.\begin{array}{llll}0.13 & 0.00 & 0.00 & 0.35\end{array}\right]$. On this data set by the establishment of the BP neural network model for fault diagnosis we can get the output layer of the network output is: $\left[\begin{array}{llllllllll}0.78 & 0.91 & 0.64 & 0.49 & 0.00 & 0.89 & 0.47 & 0.01 & 1.00 & 0.30\end{array}\right]$. Therefore we can determine the fault type of steam turbine is the ninth kind of fault, which has the same conclusion with document 3 and 4 . BP neural network training error curve as shown in Figure 1.

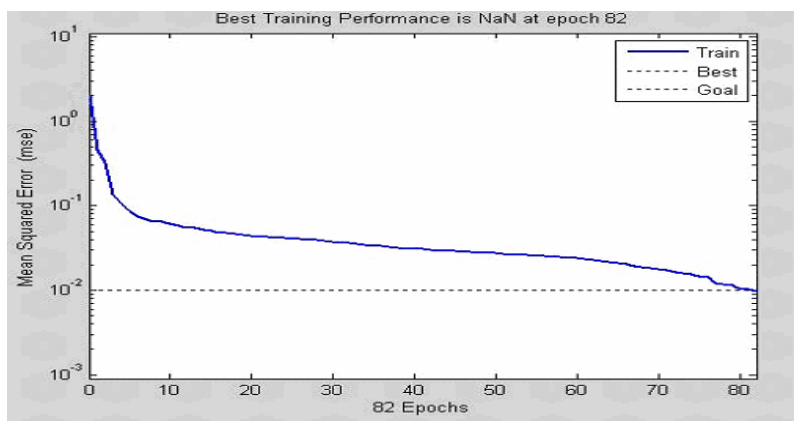

Figure 1. The training error curve of BP neural network. 
Table 3. He result of initial diagnosis of BP net and Elman net.

\begin{tabular}{cccccccccccccc}
\hline & \multicolumn{10}{c}{ Fault type } \\
\cline { 2 - 5 } & $\boldsymbol{F 1}$ & $\boldsymbol{F 2}$ & $\boldsymbol{F 3}$ & $\boldsymbol{F 4}$ & $\boldsymbol{F 5}$ & $\boldsymbol{F 6}$ & $\boldsymbol{F 7}$ & $\boldsymbol{F r}$ & $\boldsymbol{F 9}$ & $\boldsymbol{F 1 0}$ & $\boldsymbol{m}(\boldsymbol{\alpha})$ \\
\hline BP net & 0.11 & 0.13 & 0.09 & 0.07 & 0 & 0.13 & 0.07 & 0 & 0.14 & 0.04 & 0.22 \\
Elman net & 0.09 & 0.10 & 0.09 & 0.06 & 0 & 0.11 & 0.07 & 0.05 & 0.12 & 0.08 & 0.23 \\
\hline
\end{tabular}

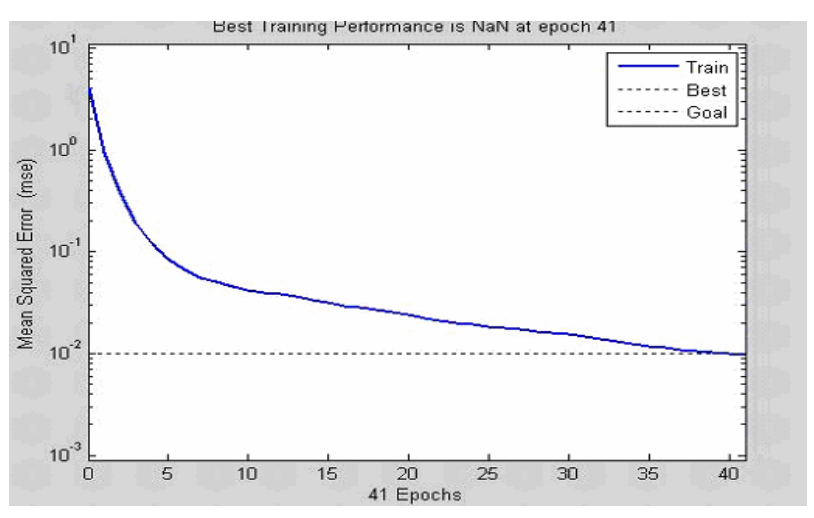

Figure 2. The training error curve of Elman neural network.

\section{Elman Neural Network Model Structure and Algorithm}

Elman neural network is a kind of feedback neural networks, which has better nonlinear fitting ability than ratio of forward neural network. Elman neural network introduced the following layer on the basis of BP neural network. As the network storage unit, it is used to store last output function. The structure of Elman neural network consisted of input layer, hidden layer, undertake layer and output layer. Compared with BP neural network, On the one hand Elman neural network's hidden layer output is delivered to the output layer neurons, on the other, transferred to undertake layer neurons to storage, during the next output in the hidden layer neurons prior to joining a hidden layer output effect. As a result of following the joining layer, Elman neural network Improve the overall stability compared these two neural network.

On the other hand, Elman neural network learning algorithm based on the improved BP algorithm, that is adaptive learning rate momentum gradient descent backpropagation algorithm. Due to it adopt this kind of algorithm, when training on the same training sample, it has better astringency than BP neural network and can also avoid network training being into local minimum .

\section{The EIman Neural Network Structure and Training}

Using the same network settings in BP neural network model, taking the data of form 1 and 2 as sample to have a training on Elman neural network. Similarly, making turbine vibration fault data of document 3 and 4 as the Elman neural network calibration sample, On this data set by the establishment of the BP neural network model for fault diagnosis we can get the output layer of the network output as shown in form Table 3. Compared the result we can find that two fault diagnosis methods are equally effective in Steam turbine fault diagnosis. Elman neural network training error curve as shown in Figure 2.

Compared Figures 1 and 2, we can see the training curve of Elman neural network model is more smooth than BP neural network. And training time is greatly reduced, reduced from 82 to 41 , significantly speeding up the convergence rate .

\section{Conclusion}

This paper uses Elman neural network learning algorithm to diagnose fault of steam turbine, compared with BP neural network, which enhance the training speed and the stability of the whole system. Through the examples of simulation validated the training and result of these two neural network, also proved that Elman neural network can achieve the anticipated target on recognizing typical type fault of Steam turbine, overcome static feedforward neural network convergence and the disadvantage of easily to fall into local minimum.

\section{REFERENCES}

[1] Sichuan Province Electric Power Industry Bureau, Sichuan Province Electric Power Education Association. Vibration of steam turbine generator units[M]. Beijing: China Electric Power Press, 1998.

[2] Jianhua Zhang, Guolian Hou, Xiaogang Sun, Guili Yuan, Using a probabilistic neural network fault diagnosis method [J], Power engineering, 2005, 25 (5):698 701

[3] Zhihong Yao. Kohonen Network in the steam turbine vibration fault diagnosis using [J]. Turbine technology, 2004, 46 (1):67 68

[4] Heji $\mathrm{Yu}$, Changzheng Chen, etc. Intelligent diagnosis based on neural network [M]. Beijing : Metallurgical Industry Press, 2000

[5] Akbari A A, et al. Induction motor identification using Elman neural network[J].WSEAS Transactions on 


\section{Systems, 2008,5:766 770}

[6] WangL, ShiX, LiangY.An improved Elman neural network with profit factors and its applications[A]. International Con-ferenceon Intelligent Computing, CIC2006[C], Aug16-19 2006, Springer Verlag, Heidelberg, D-69121,
Germany, Kun-ming, China, 2007, 317 322

[7] Huang Jinying, Pan Hongxia.The Research on Condition Detection and Fault Characteristic in one Vehicle Wheel Box. Proceedings of the 5th International Symposium on Test and Measurement,2009. 